S1 Supporting Information. Romero, Reidy, Bootsma, PreFontaine, Vryhof, Wierenga, Anderson

\title{
Synthesis of $N$-Alkenyl 2-Pyridonyl Ethers via a Au(I)-Catalyzed Rearrangement of 2-Propargyloxypyridines
}

\section{Evan O. Romero ${ }^{\prime}$, Connor P. Reidy ${ }^{I}$, Andrea N. Bootsma, Noah M. PreFontaine, Nicholas W. Vryhof, David C. Wierenga and Carolyn E. Anderson*}

Department of Chemistry and Biochemistry, Calvin College, 1726 Knollcrest Circle SE, Grand Rapids, MI 49546.

\section{Supporting Information}

Details of product ratio calculations, computational studies and references, copies of

${ }^{1} \mathrm{H}$ and ${ }^{13} \mathrm{C}$ NMR spectra for all new compounds, and HSQC and HMBC NMR spectra for compound $\mathbf{8 a}$.

\section{(22 Pages)}

Determination of Product Ratios in Crude Reaction Mixtures

S2

Computational Studies

S3-S8

${ }^{1} \mathrm{H},{ }^{13} \mathrm{C}$, HSQC, and HMBC NMR Spectra of Compound 8a

S9-S11

${ }^{1} \mathrm{H}$ and ${ }^{13} \mathrm{C}$ NMR Spectra of Compound 9

$\mathrm{S} 12$

${ }^{1} \mathrm{H}$ and ${ }^{13} \mathrm{C}$ NMR Spectra of Compound 10

$\mathrm{S} 13$

${ }^{1} \mathrm{H}$ and ${ }^{13} \mathrm{C}$ NMR Spectra of Compound 26a

S14

${ }^{1} \mathrm{H}$ and ${ }^{13} \mathrm{C}$ NMR Spectra of Compound 26b

S15

${ }^{1} \mathrm{H}$ and ${ }^{13} \mathrm{C}$ NMR Spectra of Compound 26c

S16

${ }^{1} \mathrm{H}$ and ${ }^{13} \mathrm{C}$ NMR Spectra of Compound $\boldsymbol{E}-27$

$\mathrm{S} 17$

${ }^{1} \mathrm{H}$ and ${ }^{13} \mathrm{C}$ NMR Spectra of Compound $\boldsymbol{Z}$-27

$\mathrm{S} 18$

${ }^{1} \mathrm{H}$ and ${ }^{13} \mathrm{C}$ NMR Spectra of Compound $\mathbf{2 6 f}$

${ }^{1} \mathrm{H}$ and ${ }^{13} \mathrm{C}$ NMR Spectra of Compound $\mathbf{2 6 g}$

S20

${ }^{1} \mathrm{H}$ and ${ }^{13} \mathrm{C}$ NMR Spectra of Compound $\mathbf{2 6 h}$

S21

${ }^{1} \mathrm{H}$ and ${ }^{13} \mathrm{C}$ NMR Spectra of Compound $\mathbf{8 b}$

S22

${ }^{1} \mathrm{H}$ and ${ }^{13} \mathrm{C}$ NMR Spectra of Compound $\mathbf{8 c}$

S23

${ }^{1} \mathrm{H}$ and ${ }^{13} \mathrm{C}$ NMR Spectra of Compound $\mathbf{8 d}$

S24

\footnotetext{
${ }^{1}$ Equal co-first authors
} 
S2 Supporting Information. Romero, Reidy, Bootsma, PreFontaine, Vryhof, Wierenga, Anderson

A. Determination of Product Ratios by ${ }^{1} \mathrm{H}$ NMR of Crude Reaction Mixtures

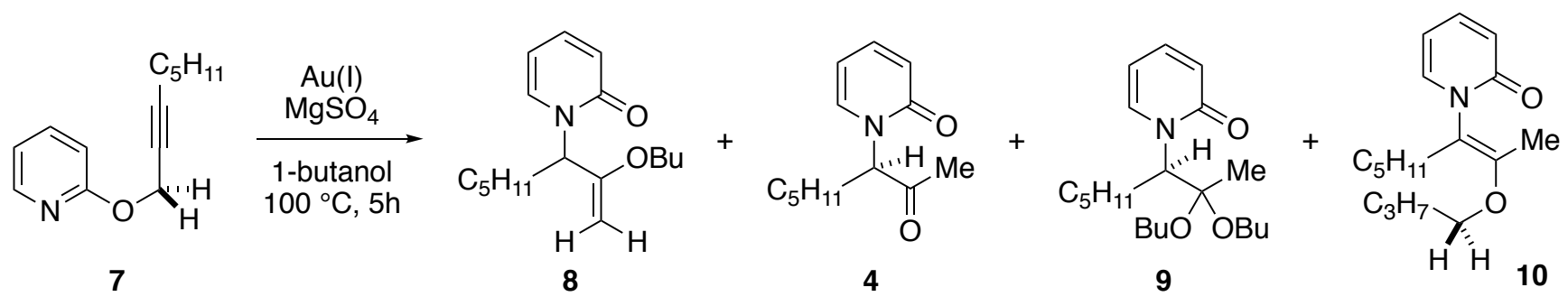

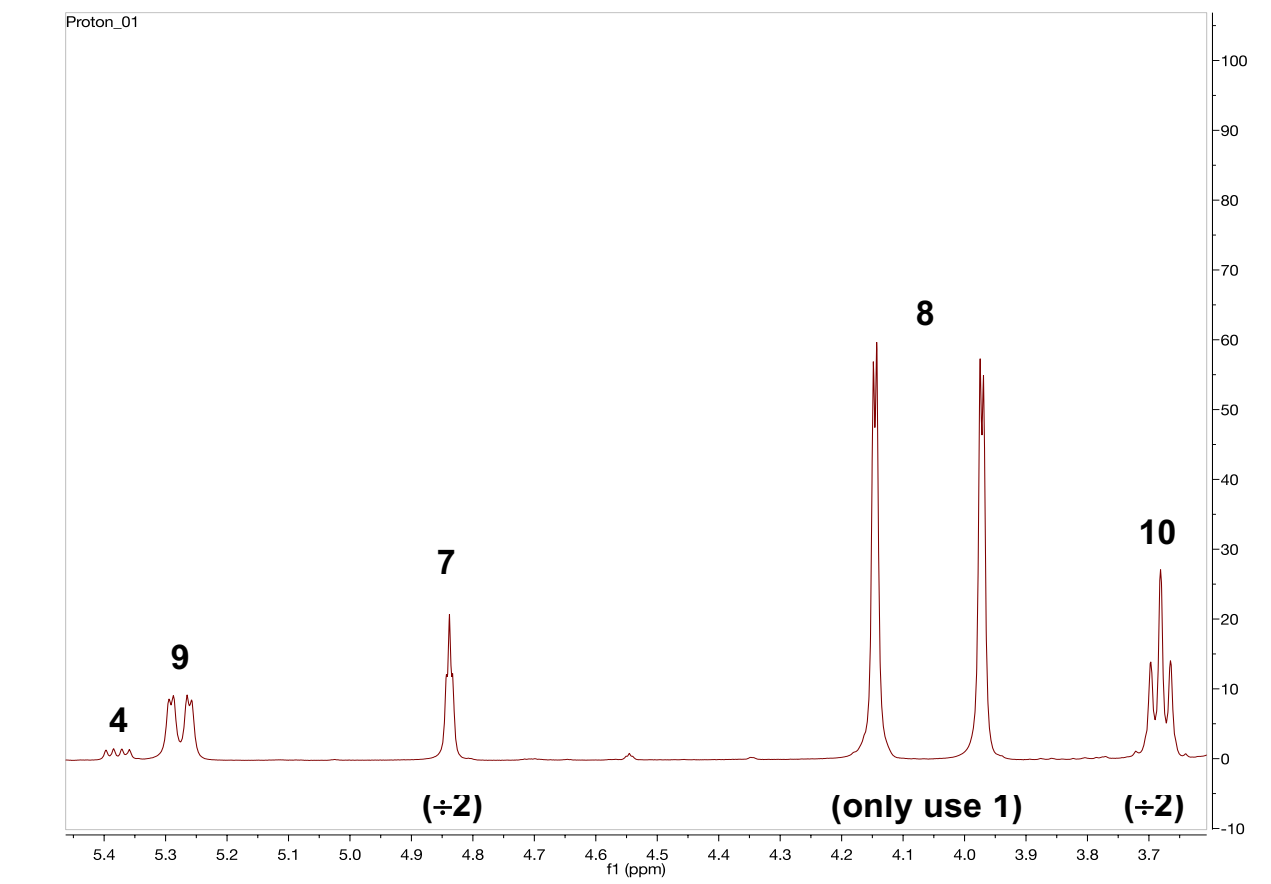

*product ratios for solvents other than 1-butanol were determined analogously.

$$
\text { selectivity ratio }=\mathbf{8} /(\mathbf{4}+\mathbf{9}+\mathbf{1 0})
$$

Product ratios were calculated by direct comparison of the integration of each resonance, taking into account the number of protons represented by each group (e.g. the integration for the resonances for compound 7 and $\mathbf{1 0}$ were each divided by 2 and only one of the peaks for compound $\mathbf{8}$ was considered when determining the overall ratios.) The following experiment suggests that for ether $\mathbf{8}$ and ketone $\mathbf{4}$, direct comparison should be accurate to within $2 \%$.<smiles>CCCCCCC(=CC=[18O])n1ccccc1=O</smiles>

experimentally measured observed by ${ }^{1} \mathrm{H}$ NMR

$0.044 \mathrm{mmol}$

$0.045 \mathrm{mmol}$<smiles>CC(=O)C([14CH3])n1ccccc1=O</smiles>

$0.051 \mathrm{mmol}$

$0.052 \mathrm{mmol}$ 
S3 Supporting Information. Romero, Reidy, Bootsma, PreFontaine, Vryhof, Wierenga, Anderson

\section{B. Computational Studies ${ }^{1}$}

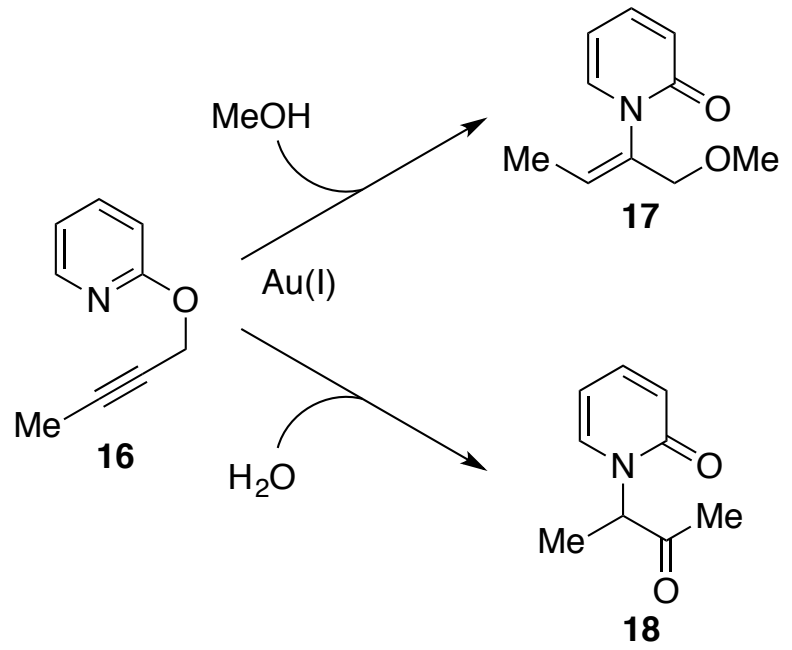

\section{Background to Computational Study}

All calculations were performed with the Gaussian09 ab initio quantum chemistry program. ${ }^{1}$ The geometries of all compounds were optimized with [B3LYP/M062X] using a basis set of 6-31G(d) for all atoms. Analytical vibrational frequencies were computed with the 6-31G(d) basis to confirm proper convergence to well-defined energy minima. The energies of the optimized structures were reevaluated by additional single point calculations on each optimized geometry using the $6-311+\mathrm{G}(2 \mathrm{~d}, \mathrm{p})$ basis set. In this way, the accuracy of the higher level of theory was obtained without the computational costs associated with performing the entire optimization and frequency calculations at the higher level of theory. The Free Energie,s $\mathbf{G}(6-311+\mathrm{G}(2 \mathrm{~d}, \mathrm{p}))$, used to calculate the energy differences reported in this article have been computed with the following protocol:

$$
\begin{aligned}
& \mathbf{G}(6-311+\mathrm{G}(2 \mathrm{~d}, \mathrm{p}))=\mathbf{E}(6-311+\mathrm{G}(2 \mathrm{~d}, \mathrm{p})+\mathbf{T C} \\
& \mathbf{T C}=\mathbf{G}(6-31 \mathrm{G}(\mathrm{d}))-\mathbf{E}(6-31 \mathrm{G}(\mathrm{d}))
\end{aligned}
$$

Where $\mathbf{E}(\mathrm{x})$ is the SCF energy in the $6-31 \mathrm{G}(\mathrm{d})$ or $6-311+\mathrm{G}(2 \mathrm{~d}, \mathrm{p})$ basis sets and TC is the thermal correction to the energy calculated for the $6-31 \mathrm{G}(\mathrm{d})$ basis set. $\mathbf{G}(6-311+\mathrm{G}(2 \mathrm{~d}, \mathrm{p}))$ is the free energy at $298.15 \mathrm{~K}$ for the $6-31 \mathrm{G}(\mathrm{d})$ basis set.

1. Gaussian 09, Revision B.01, M. J. Frisch, G. W. Trucks, H. B. Schlegel, G. E. Scuseria, M. A. Robb, J. R. Cheeseman, G. Scalmani, V. Barone, B. Mennucci, G. A. Petersson, H. Nakatsuji, M. Caricato, X. Li, H. P. Hratchian, A. F. Izmaylov, J. Bloino, G. Zheng, J. L. Sonnenberg, M. Hada, M. Ehara, K. Toyota, R. Fukuda, J. Hasegawa, M. Ishida, T. Nakajima, Y. Honda, O. Kitao, H. Nakai, T. Vreven, J. A. Montgomery, Jr., J. E. Peralta, F. Ogliaro, M. Bearpark, J. J. Heyd, E. Brothers, K. N. Kudin, V. N. Staroverov, T. Keith, R. Kobayashi, J. Normand, K. Raghavachari, A. Rendell, J. C. Burant, S. S. Iyengar, J. Tomasi, M. Cossi, N. Rega, J. M. Millam, M. Klene, J. E. Knox, J. B. Cross, V. Bakken, C. Adamo, J. Jaramillo, R. Gomperts, R. E. Stratmann, O. Yazyev, A. J. Austin, R. Cammi, C. Pomelli, J. W. Ochterski, R. L. Martin, K. Morokuma, V. G. Zakrzewski, G. A. Voth, P. Salvador, J. J. Dannenberg, S. Dapprich, A. D. Daniels, O. Farkas, J. B. Foresman, J. V. Ortiz, J. Cioslowski, and D. J. Fox, Gaussian, Inc., Wallingford CT, 2010. 
S4 Supporting Information. Romero, Reidy, Bootsma, PreFontaine, Vryhof, Wierenga, Anderson

SCF energies and Gibbs free energies of calculated structures.

Table 1: Energies calculated at the B3LYP level of theory

\begin{tabular}{ccccc}
\hline & $\begin{array}{c}\mathbf{E}(6-31 \mathrm{G}(\mathrm{d})) \\
\text { hartree }\end{array}$ & $\begin{array}{c}\mathbf{G}(6-31 \mathrm{G}(\mathrm{d})) \\
\text { hartree }\end{array}$ & $\begin{array}{c}\mathbf{E}(6-311+\mathrm{G}(2 \mathrm{~d}, \mathrm{p})) \\
\text { hartree }\end{array}$ & $\begin{array}{c}\mathbf{G}(6-311+\mathrm{G}(2 \mathrm{~d}, \mathrm{p})) \\
\text { hartree }\end{array}$ \\
\hline $\mathbf{1 6}$ & -478.28165 & -478.15868 & -478.42616 & -478.30319 \\
$\mathbf{1 7}$ & -594.05609 & -593.88086 & -594.24058 & -594.06535 \\
$\mathbf{1 8}$ & -554.78479 & -554.63592 & -554.95586 & -554.80700 \\
$\mathrm{H}_{2} \mathrm{O}$ & -76.40895 & -76.40611 & -76.45942 & -76.45658 \\
$\mathrm{MeOH}$ & -115.71441 & -115.68566 & -115.76745 & -115.73870 \\
\hline $\mathbf{1 7}-(\mathbf{1 6}+\mathrm{MeOH})$ & -0.06003 & -0.03652 & -0.04697 & -0.02346 \\
$(\mathrm{kcal} / \mathrm{mol})$ & $(-37.7)$ & $(-22.9)$ & $(-29.5)$ & $(-14.7)$ \\
\hline $\mathbf{1 8}-\left(\mathbf{1 6}+\mathrm{H}_{2} \mathrm{O}\right)$ & -0.09419 & -0.07113 & -0.07028 & -0.04723 \\
$(\mathrm{kcal} / \mathrm{mol})$ & $(-59.1)$ & $(-44.6)$ & $(-44.1)$ & $(-29.6)$ \\
\hline$\Delta(\mathrm{kcal} / \mathrm{mol})$ & 21.4 & 21.7 & 14.6 & 14.9 \\
\hline
\end{tabular}

Table 2: Energies calculated at the M06-2X level of theory

\begin{tabular}{ccccc}
\hline & $\begin{array}{c}\mathbf{E}(6-31 \mathrm{G}(\mathrm{d})) \\
\text { hartree }\end{array}$ & $\begin{array}{c}\mathbf{G}(6-31 \mathrm{G}(\mathrm{d})) \\
\text { hartree }\end{array}$ & $\begin{array}{c}\mathbf{E}(6-311+\mathrm{G}(2 \mathrm{~d}, \mathrm{p})) \\
\text { hartree }\end{array}$ & $\begin{array}{c}\mathbf{G}(6-311+\mathrm{G}(2 \mathrm{~d}, \mathrm{p})) \\
\text { hartree }\end{array}$ \\
\hline $\mathbf{1 6}$ & -478.07520 & -477.94975 & -478.22264 & -478.09719 \\
$\mathbf{1 7}$ & -593.79696 & -593.61877 & -593.98558 & -593.80738 \\
$\mathbf{1 8}$ & -554.54654 & -554.39463 & -554.72176 & -554.56984 \\
$\mathrm{H}_{2} \mathrm{O}$ & -76.37339 & -76.37017 & -76.42198 & -76.41876 \\
$\mathrm{MeOH}$ & -115.65476 & -115.62532 & -115.70693 & -115.67749 \\
\hline $\mathbf{1 7}-(\mathbf{1 6}+\mathrm{MeOH})$ & -0.067 & -0.0437 & -0.05601 & -0.0327 \\
$(\mathrm{kcal} / \mathrm{mol})$ & $(-42.0)$ & $(-27.4)$ & $(-35.1)$ & $(-20.5)$ \\
\hline $\mathbf{1 8}-\left(\mathbf{1 6}+\mathrm{H}_{2} \mathrm{O}\right)$ & -0.09795 & -0.07471 & -0.07714 & -0.05389 \\
$(\mathrm{kcal} / \mathrm{mol})$ & $(-61.5)$ & $(-46.9)$ & $(-48.4)$ & $(-33.8)$ \\
\hline$\Delta(\mathrm{kcal} / \mathrm{mol})$ & 19.4 & 19.5 & 13.3 & 13.3 \\
\hline
\end{tabular}

\section{Cartesian coordinates of the optimized structures.}

\section{6 (B3LYP)}

$\begin{array}{llll}\mathrm{C} & 0.00000000 & 0.00000000 & 0.00000000 \\ \mathrm{O} & 0.83784200 & 1.17437700 & -0.00009000 \\ \mathrm{C} & 2.17470000 & 0.96222400 & -0.00019900 \\ \mathrm{C} & 2.99273000 & 2.10796300 & -0.00018700 \\ \mathrm{C} & 4.36444600 & 1.91770100 & -0.00033600 \\ \mathrm{C} & 4.87986100 & 0.61324100 & -0.00049300 \\ \mathrm{C} & 3.97701700 & -0.44119200 & -0.00050800 \\ \mathrm{~N} & 2.64202100 & -0.28173400 & -0.00037400 \\ \mathrm{H} & 4.32691800 & -1.47210200 & -0.00063700 \\ \mathrm{H} & 5.94868300 & 0.42591800 & -0.00060600 \\ \mathrm{H} & 5.03225900 & 2.77514100 & -0.00032800 \\ \mathrm{H} & 2.53845600 & 3.09258700 & -0.00006900\end{array}$


S5 Supporting Information. Romero, Reidy, Bootsma, PreFontaine, Vryhof, Wierenga, Anderson

$\begin{array}{llll}\mathrm{C} & -1.39503400 & 0.42456200 & 0.00042000 \\ \mathrm{C} & -2.56192200 & 0.73911100 & -0.00026900 \\ \mathrm{C} & -3.96984100 & 1.12647500 & -0.00074800 \\ \mathrm{H} & -4.62040000 & 0.24344400 & -0.00410600 \\ \mathrm{H} & -4.22065100 & 1.72000000 & 0.88660500 \\ \mathrm{H} & -4.21851400 & 1.72506600 & -0.88529700 \\ \mathrm{H} & 0.23159200 & -0.61194800 & -0.88006000 \\ \mathrm{H} & 0.23231000 & -0.61232200 & 0.87960500\end{array}$

\section{6 (M06-2X)}

$\begin{array}{llll}\mathrm{C} & 0.00000000 & 0.00000000 & 0.00000000 \\ \mathrm{O} & 0.81914300 & 1.16952400 & -0.00000100 \\ \mathrm{C} & 2.15055600 & 0.95879300 & -0.00000200 \\ \mathrm{C} & 2.96510900 & 2.10370600 & 0.00003200 \\ \mathrm{C} & 4.33188000 & 1.91403400 & 0.00002800 \\ \mathrm{C} & 4.84572500 & 0.61148800 & -0.00000500 \\ \mathrm{C} & 3.94523700 & -0.43892700 & -0.00003200 \\ \mathrm{~N} & 2.61317600 & -0.28001500 & -0.00003100 \\ \mathrm{H} & 4.29369800 & -1.46895600 & -0.00005700 \\ \mathrm{H} & 5.91293500 & 0.42334300 & -0.00000900 \\ \mathrm{H} & 4.99978400 & 2.77013000 & 0.00005200 \\ \mathrm{H} & 2.50537700 & 3.08470400 & 0.00005800 \\ \mathrm{C} & -1.40046500 & 0.42003700 & 0.00003100 \\ \mathrm{C} & -2.56370900 & 0.73542700 & -0.00001700 \\ \mathrm{C} & -3.97329100 & 1.12495700 & -0.00022600 \\ \mathrm{H} & -4.61768000 & 0.24118400 & -0.00339500 \\ \mathrm{H} & -4.21486000 & 1.71688700 & 0.88697700 \\ \mathrm{H} & -4.21314000 & 1.72196800 & -0.88449100 \\ \mathrm{H} & 0.22912500 & -0.60992800 & -0.88046900 \\ \mathrm{H} & 0.22916800 & -0.60994600 & 0.88044500\end{array}$

\section{7 (B3LYP)}

$\begin{array}{llll}\mathrm{C} & 0.00000000 & 0.00000000 & 0.00000000 \\ \mathrm{C} & 1.12345700 & -0.93168800 & 0.34111400 \\ \mathrm{C} & 1.04882700 & -2.24815000 & 0.55571200 \\ \mathrm{~N} & -0.19426700 & -2.96720200 & 0.39059500 \\ \mathrm{C} & -0.61408900 & -3.30109800 & -0.87023400 \\ \mathrm{C} & -1.76445300 & -4.00439900 & -1.07881300 \\ \mathrm{C} & -2.52270400 & -4.40747200 & 0.05650500 \\ \mathrm{C} & -2.10443400 & -4.10585100 & 1.32117200 \\ \mathrm{C} & -0.88203300 & -3.36975500 & 1.57426000 \\ \mathrm{O} & -0.42639100 & -3.08033000 & 2.67901300 \\ \mathrm{H} & -2.66052400 & -4.40835800 & 2.20169700 \\ \mathrm{H} & -3.44355400 & -4.96771000 & -0.08844000 \\ \mathrm{H} & -2.07443300 & -4.25108900 & -2.08713900 \\ \mathrm{H} & 0.03555500 & -2.97076800 & -1.67204700 \\ \mathrm{C} & 2.21959900 & -3.10340700 & 0.95057800 \\ \mathrm{O} & 2.47750700 & -4.02513200 & -0.10104900 \\ \mathrm{C} & 3.43269900 & -5.00254400 & 0.25694400\end{array}$


S6 Supporting Information. Romero, Reidy, Bootsma, PreFontaine, Vryhof, Wierenga, Anderson

$\begin{array}{llll}\mathrm{H} & 3.10762500 & -5.58839000 & 1.13131000 \\ \mathrm{H} & 3.54401500 & -5.67208400 & -0.60001900 \\ \mathrm{H} & 4.41222000 & -4.55254800 & 0.48702900 \\ \mathrm{H} & 3.09946200 & -2.46840400 & 1.14099800 \\ \mathrm{H} & 1.97997300 & -3.63852400 & 1.88169700 \\ \mathrm{H} & 2.10856300 & -0.47870300 & 0.44494800 \\ \mathrm{H} & -0.06482900 & 0.80276500 & 0.74551800 \\ \mathrm{H} & 0.17407100 & 0.48439200 & -0.97000100 \\ \mathrm{H} & -0.96579600 & -0.50941700 & -0.03734100\end{array}$

\section{7 (M06-2X)}

$\begin{array}{llll}\mathrm{C} & 0.00000000 & 0.00000000 & 0.00000000 \\ \mathrm{C} & 1.15772400 & -0.90263400 & 0.29525600 \\ \mathrm{C} & 1.10582900 & -2.22167500 & 0.46403100 \\ \mathrm{~N} & -0.12513400 & -2.94431000 & 0.28334300 \\ \mathrm{C} & -0.58993000 & -3.16184600 & -0.98380700 \\ \mathrm{C} & -1.73696900 & -3.85204900 & -1.21283700 \\ \mathrm{C} & -2.44251800 & -4.36832800 & -0.08717400 \\ \mathrm{C} & -1.97722600 & -4.18267000 & 1.17603900 \\ \mathrm{C} & -0.75210200 & -3.45268200 & 1.44314100 \\ \mathrm{O} & -0.25986900 & -3.25329000 & 2.54475800 \\ \mathrm{H} & -2.49067400 & -4.56973100 & 2.04817400 \\ \mathrm{H} & -3.36307400 & -4.92341000 & -0.24513200 \\ \mathrm{H} & -2.08593900 & -4.00800900 & -2.22492500 \\ \mathrm{H} & 0.02882400 & -2.74627800 & -1.77114700 \\ \mathrm{C} & 2.28148700 & -3.09054900 & 0.79312200 \\ \mathrm{O} & 2.47668600 & -3.97589100 & -0.28858100 \\ \mathrm{C} & 3.44126600 & -4.95570800 & 0.00213500 \\ \mathrm{H} & 3.14783500 & -5.55924800 & 0.87290900 \\ \mathrm{H} & 3.52181900 & -5.60277400 & -0.87291100 \\ \mathrm{H} & 4.42366700 & -4.50474100 & 0.20640700 \\ \mathrm{H} & 3.17376800 & -2.46802200 & 0.96014000 \\ \mathrm{H} & 2.06959100 & -3.64923200 & 1.71669700 \\ \mathrm{H} & 2.13492700 & -0.43441200 & 0.39640100 \\ \mathrm{H} & -0.05214800 & 0.80104000 & 0.74425900 \\ \mathrm{H} & 0.11892100 & 0.47839600 & -0.97854200 \\ \mathrm{H} & -0.94899500 & -0.54005300 & 0.00894800\end{array}$

\section{8 (B3LYP)}

$\begin{array}{llll}\mathrm{C} & 0.00000000 & 0.00000000 & 0.00000000 \\ \mathrm{~N} & -1.39693500 & -0.19297000 & -0.45981300 \\ \mathrm{C} & -1.80483200 & -1.41911200 & -0.91393700 \\ \mathrm{C} & -3.08844400 & -1.64668300 & -1.31630000 \\ \mathrm{C} & -4.01485500 & -0.56872700 & -1.25936000 \\ \mathrm{C} & -3.62030000 & 0.66706600 & -0.83232600 \\ \mathrm{C} & -2.25789300 & 0.94517100 & -0.43097900 \\ \mathrm{O} & -1.82369400 & 2.04683300 & -0.08936700 \\ \mathrm{H} & -4.30150500 & 1.50950900 & -0.78965400 \\ \mathrm{H} & -5.04543900 & -0.73134900 & -1.56544100\end{array}$


S7 Supporting Information. Romero, Reidy, Bootsma, PreFontaine, Vryhof, Wierenga, Anderson

$\begin{array}{llll}\mathrm{H} & -3.37792700 & -2.62852000 & -1.67100800 \\ \mathrm{H} & -1.03754800 & -2.18367900 & -0.94535000 \\ \mathrm{C} & 0.40487600 & -0.98835600 & 1.09125500 \\ \mathrm{H} & 1.38811400 & -0.71087300 & 1.48558400 \\ \mathrm{H} & -0.31557700 & -0.96295700 & 1.91438200 \\ \mathrm{H} & 0.48494100 & -2.00950600 & 0.70991000 \\ \mathrm{C} & 0.90823000 & -0.08518900 & -1.24822700 \\ \mathrm{C} & 1.10374300 & 1.19850900 & -2.02247600 \\ \mathrm{H} & 0.16805000 & 1.76321700 & -2.09380700 \\ \mathrm{H} & 1.80953900 & 1.83722800 & -1.47330100 \\ \mathrm{H} & 1.51366700 & 0.98761900 & -3.01232200 \\ \mathrm{O} & 1.40658900 & -1.14421800 & -1.57931600 \\ \mathrm{H} & 0.00879800 & 1.02204100 & 0.38355000\end{array}$

\section{8 (M06-2X)}

$\begin{array}{llll}\mathrm{C} & 0.00000000 & 0.00000000 & 0.00000000 \\ \mathrm{~N} & -1.39157500 & -0.17396500 & -0.45838100 \\ \mathrm{C} & -1.78228300 & -1.37939600 & -0.97244800 \\ \mathrm{C} & -3.05612300 & -1.60398600 & -1.38563700 \\ \mathrm{C} & -3.99376600 & -0.53736200 & -1.27526600 \\ \mathrm{C} & -3.61353400 & 0.67572300 & -0.79574600 \\ \mathrm{C} & -2.24945100 & 0.94682300 & -0.38704500 \\ \mathrm{O} & -1.82429000 & 2.03308500 & -0.01151500 \\ \mathrm{H} & -4.29900200 & 1.51054300 & -0.71265100 \\ \mathrm{H} & -5.02247000 & -0.69594800 & -1.58604200 \\ \mathrm{H} & -3.33410700 & -2.56872200 & -1.78871000 \\ \mathrm{H} & -1.00167600 & -2.12975900 & -1.03794900 \\ \mathrm{C} & 0.42983800 & -1.07294700 & 0.98712100 \\ \mathrm{H} & 1.40932900 & -0.81311000 & 1.39693100 \\ \mathrm{H} & -0.28679200 & -1.14051700 & 1.80907400 \\ \mathrm{H} & 0.53000100 & -2.04785200 & 0.50583400 \\ \mathrm{C} & 0.86922200 & 0.01977500 & -1.26741800 \\ \mathrm{C} & 0.89203000 & 1.32105100 & -2.02869300 \\ \mathrm{H} & -0.08796900 & 1.80659800 & -2.00431200 \\ \mathrm{H} & 1.59122900 & 2.00038000 & -1.52636100 \\ \mathrm{H} & 1.23037600 & 1.15499400 & -3.05175300 \\ \mathrm{O} & 1.45804200 & -0.97479400 & -1.62446500 \\ \mathrm{H} & 0.01301400 & 0.98906600 & 0.46261100\end{array}$

\section{Water (B3LYP)}

$\begin{array}{llll}\mathrm{O} & 0.00000000 & 0.00000000 & 0.00000000 \\ \mathrm{H} & 0.76147400 & -0.59872000 & 0.00000000 \\ \mathrm{H} & -0.76147400 & -0.59871900 & 0.00000000\end{array}$

$\begin{array}{llll}\text { Water (M06-2X) } & & \\ \mathrm{O} & 0.00000000 & 0.00000000 & 0.00000000 \\ \mathrm{H} & 0.76228400 & -0.59246200 & 0.00000000 \\ \mathrm{H} & -0.76228400 & -0.59246200 & 0.00000000\end{array}$


S8 Supporting Information. Romero, Reidy, Bootsma, PreFontaine, Vryhof, Wierenga, Anderson

\begin{tabular}{llll}
\multicolumn{4}{l}{ Methanol (B3LYP) } \\
$\mathrm{C}$ & 0.00000000 & 0.00000000 & 0.00000000 \\
$\mathrm{O}$ & 0.00000000 & -1.41882800 & 0.00000000 \\
$\mathrm{H}$ & 0.92312900 & -1.71289400 & 0.00000000 \\
$\mathrm{H}$ & -1.04733500 & 0.31419500 & 0.00000000 \\
$\mathrm{H}$ & 0.48402100 & 0.42517500 & 0.89314000 \\
$\mathrm{H}$ & 0.48402100 & 0.42517500 & -0.89314000
\end{tabular}

Methanol (M06-2X)

$\begin{array}{llll}\text { C } & 0.00000000 & 0.00000000 & 0.00000000\end{array}$

O $\quad 0.00000000 \quad-1.41092300 \quad 0.00000000$

$\mathrm{H} \quad 0.91840400 \quad-1.70951300 \quad 0.00000000$

$\begin{array}{llll}\mathrm{H} & -1.04499500 & 0.31545900 & 0.00000000\end{array}$

$\mathrm{H} \quad 0.48397300 \quad 0.42039200 \quad 0.89192000$

$\mathrm{H} \quad 0.48397300 \quad 0.42039200 \quad-0.89192000$ 
S9 Supporting Information. Romero, Reidy, Bootsma, PreFontaine, Vryhof, Wierenga, Anderson C. NMR Spectra

\section{Compound 8a}
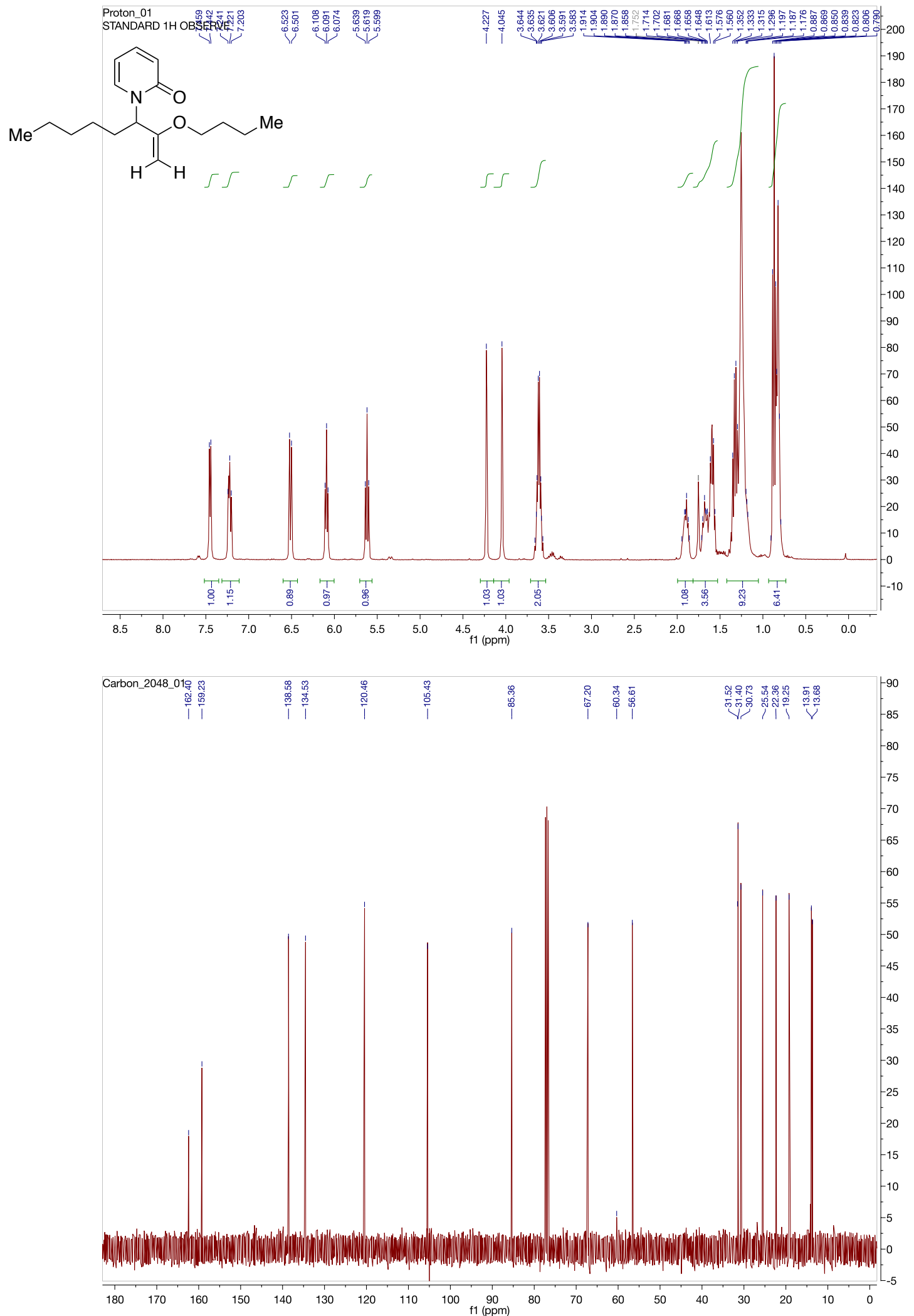
S10 Supporting Information. Romero, Reidy, Bootsma, PreFontaine, Vryhof, Wierenga, Anderson

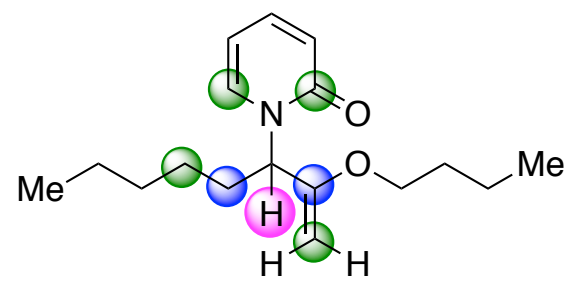

\begin{tabular}{|c|c|}
\hline $\begin{array}{l}=\mathrm{H} \text { responsible } \\
\text { for the }{ }^{1} \mathrm{H} \text { signal at } \\
\\
5.62 \mathrm{ppm} \text {, which }\end{array}$ & $\begin{aligned} \mathrm{O}= & 2 \text { bond neighbors } \\
& \text { (observed in } 8 \mathrm{~Hz} \\
& \text { HBMC) }\end{aligned}$ \\
\hline
\end{tabular}

correlates to the

${ }^{13} \mathrm{C}$ signal at 56.6

ppm (HSQC)

HSQC

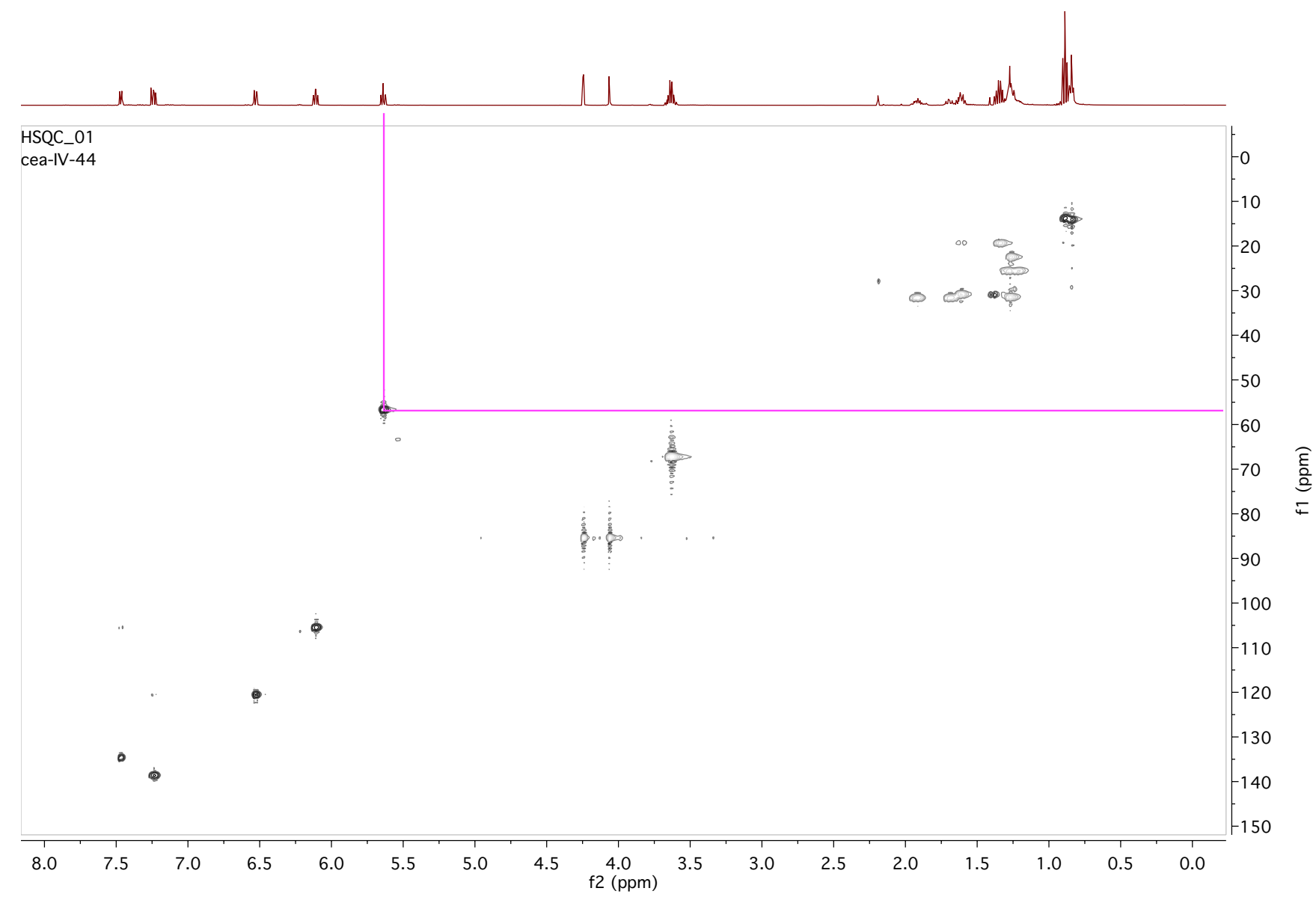


S11 Supporting Information. Romero, Reidy, Bootsma, PreFontaine, Vryhof, Wierenga, Anderson $\operatorname{HMBC}(8 \mathrm{~Hz})$

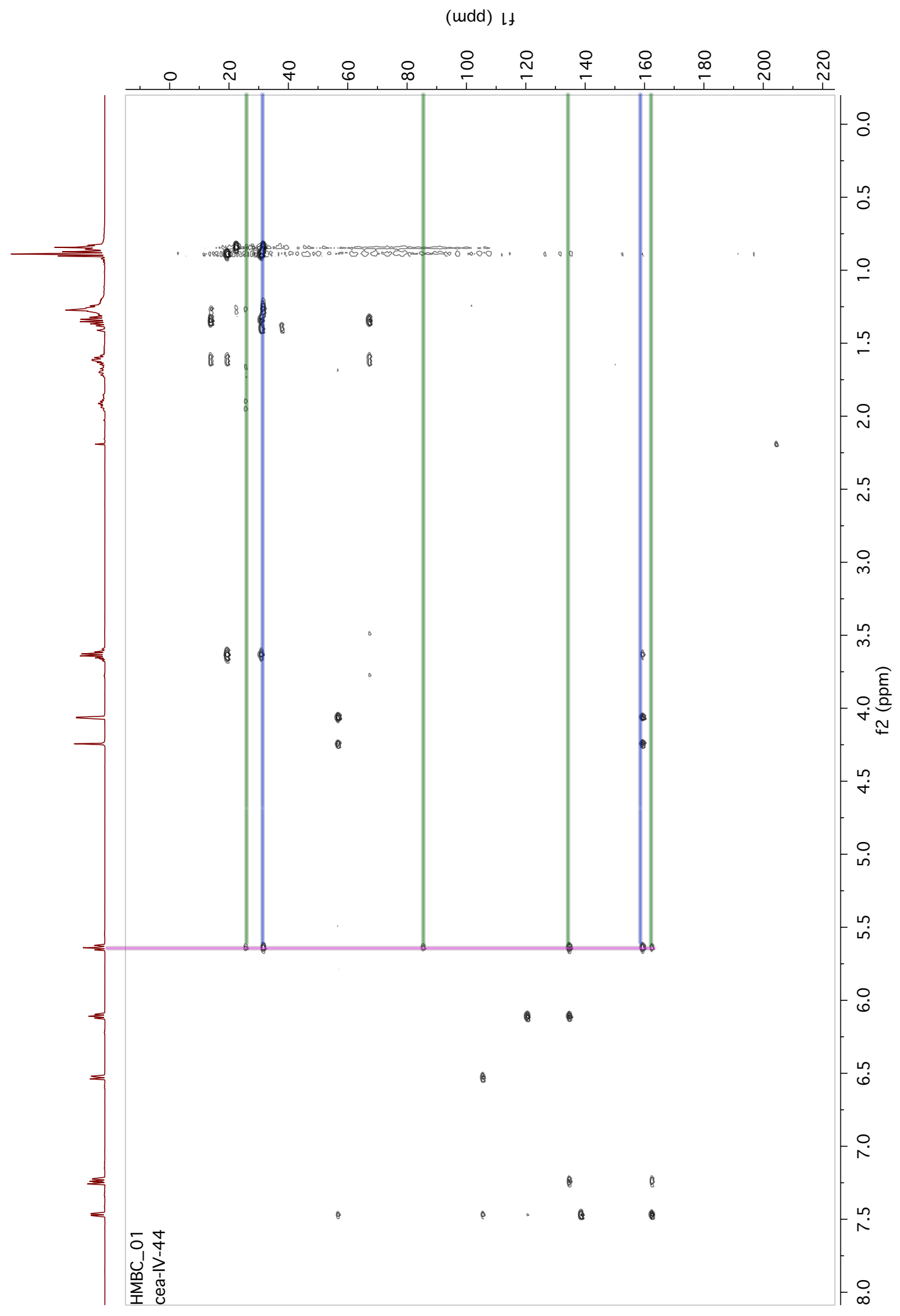


S12 Supporting Information. Romero, Reidy, Bootsma, PreFontaine, Vryhof, Wierenga, Anderson

\section{Compound 9}
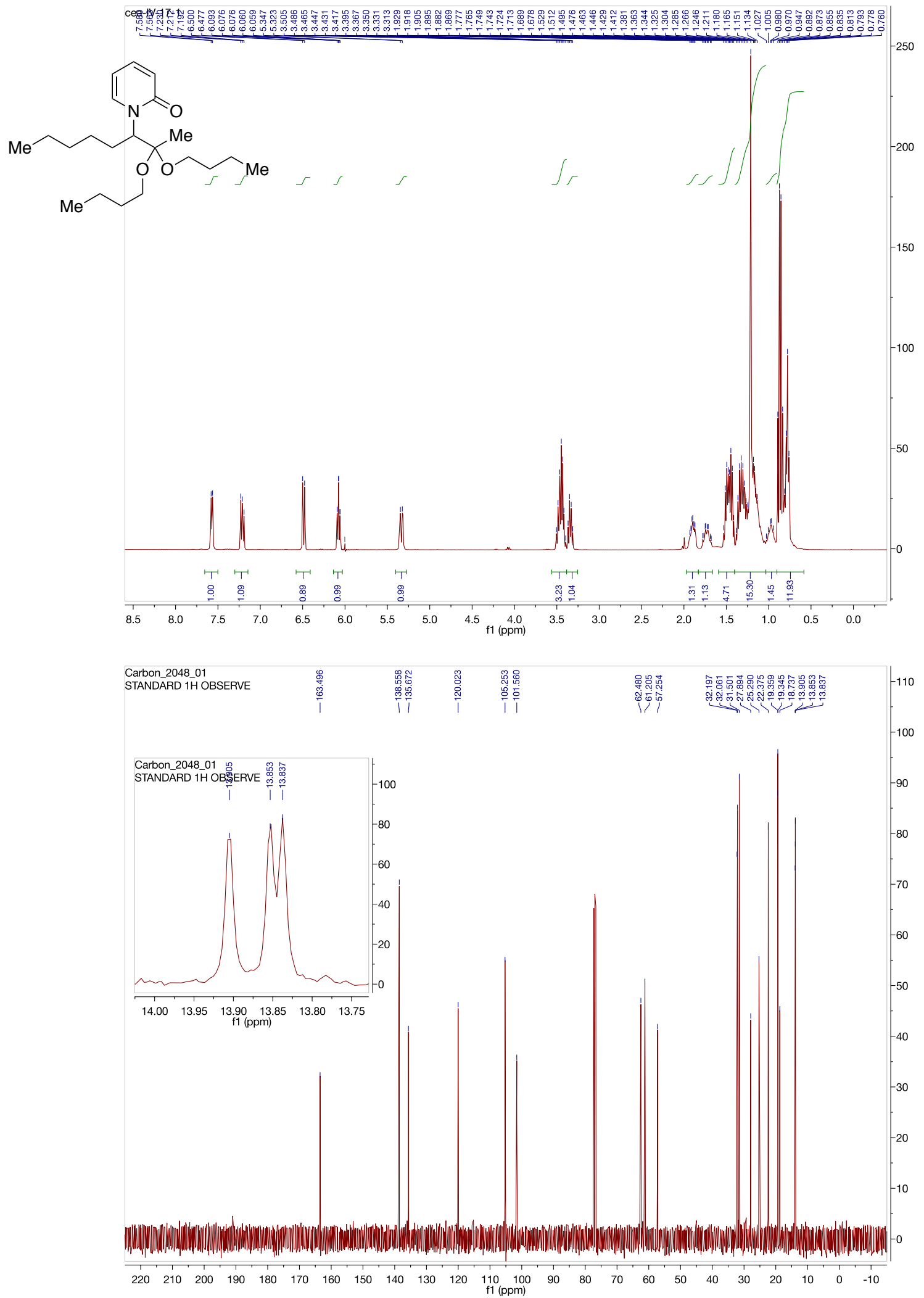
S13 Supporting Information. Romero, Reidy, Bootsma, PreFontaine, Vryhof, Wierenga, Anderson

Compound 10 (isolated as 5:1 mixture with ketone 4)
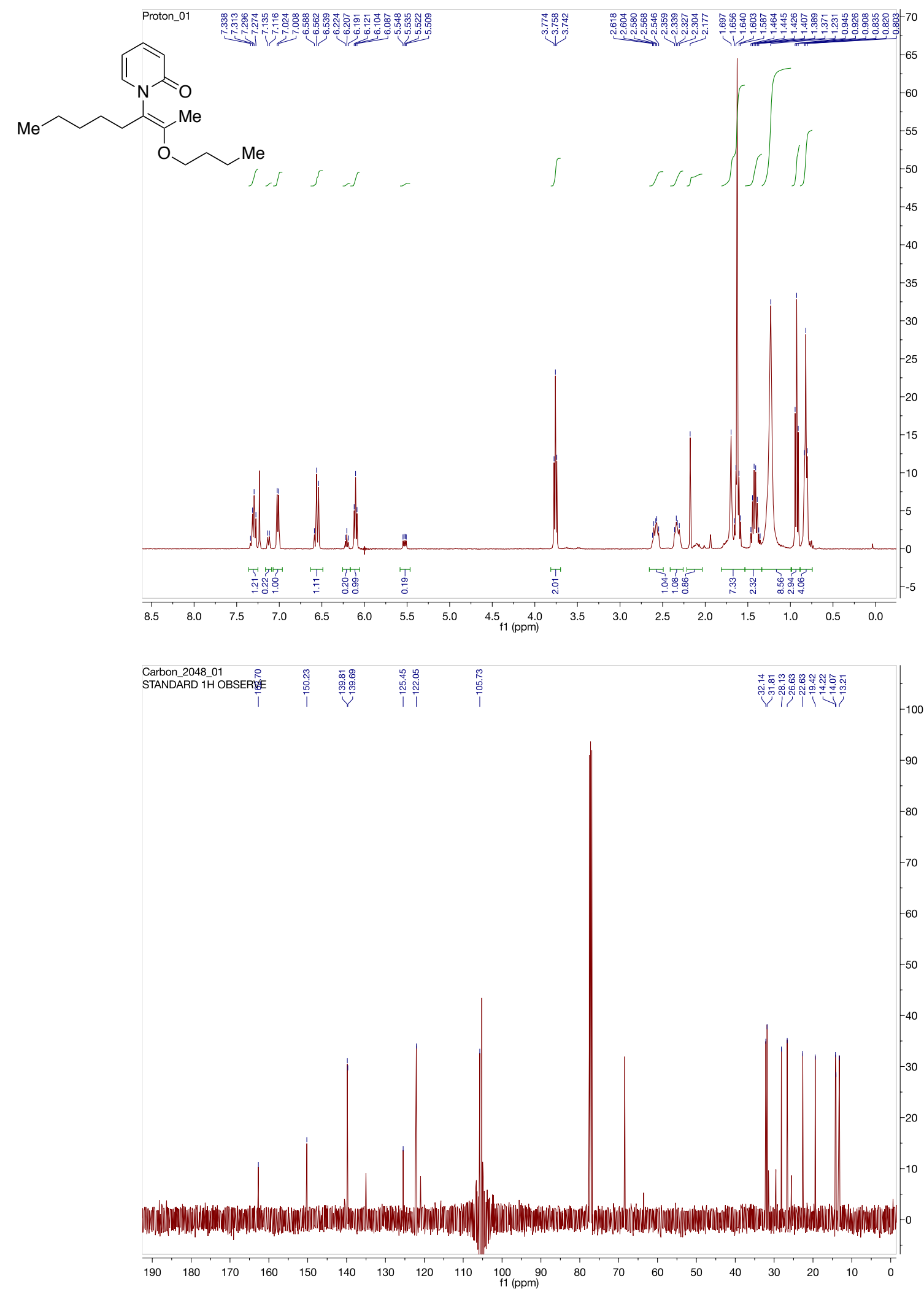
S14 Supporting Information. Romero, Reidy, Bootsma, PreFontaine, Vryhof, Wierenga, Anderson

\section{Compound 26a}
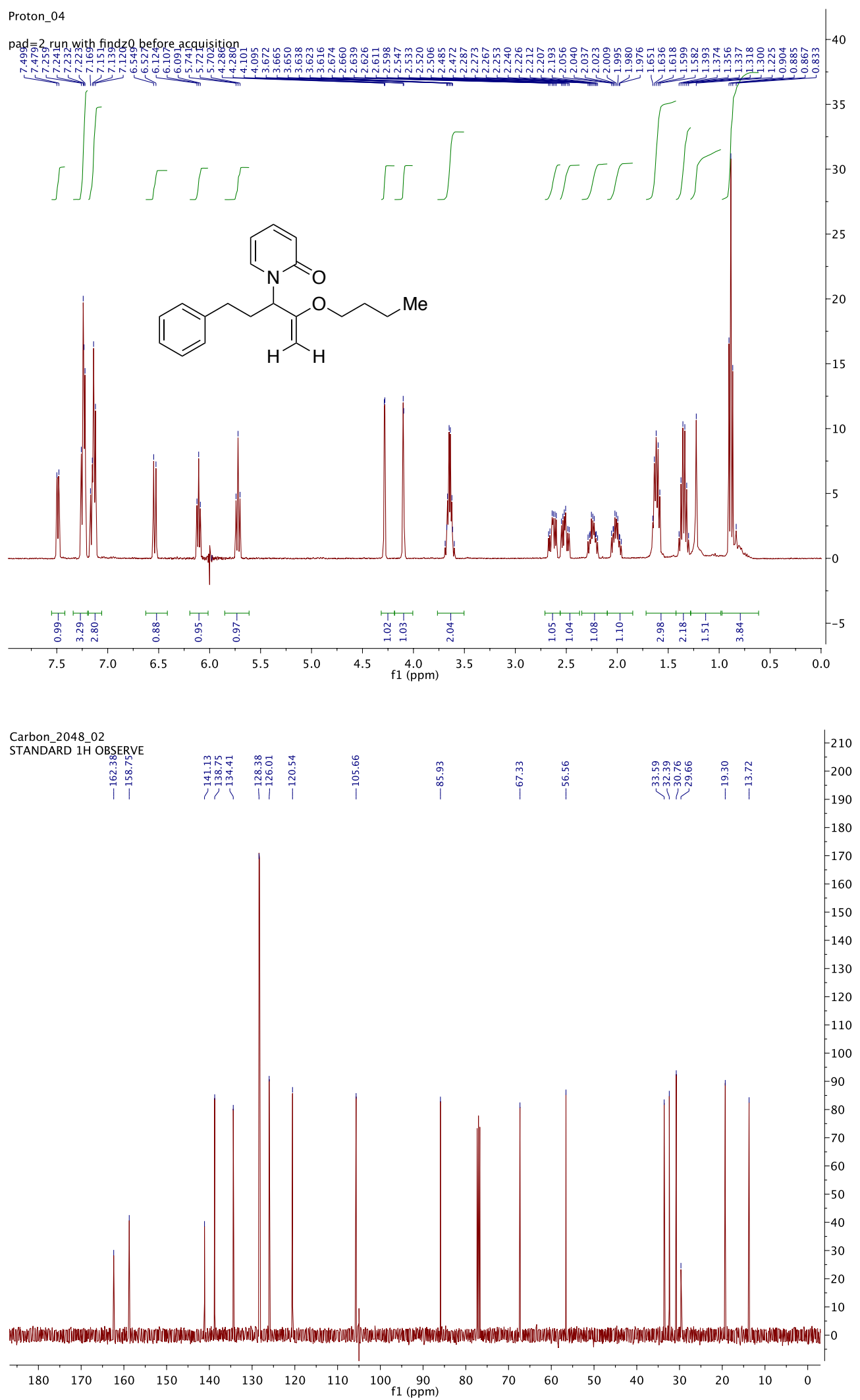
S15 Supporting Information. Romero, Reidy, Bootsma, PreFontaine, Vryhof, Wierenga, Anderson

\section{Compound 26b}
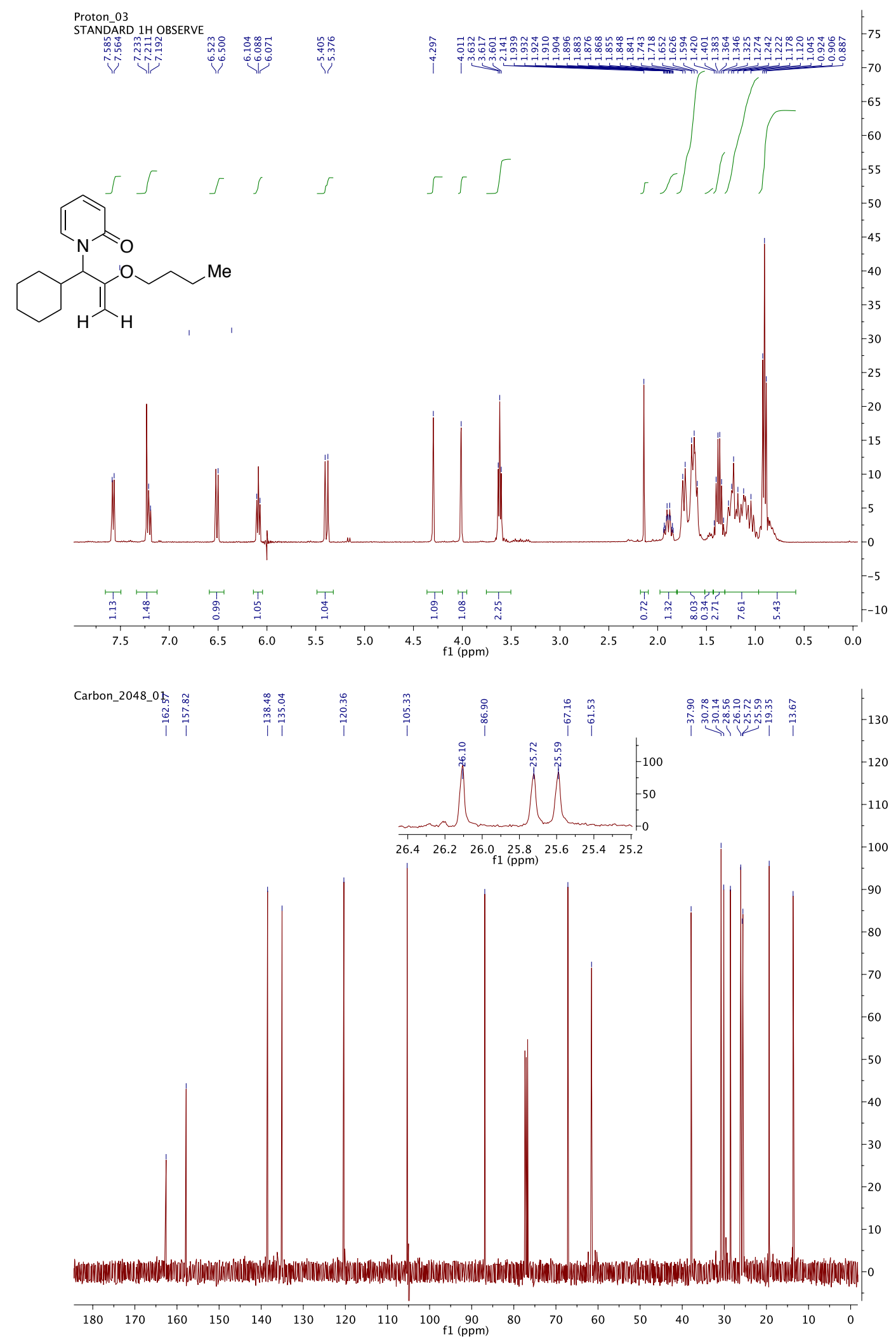
S16 Supporting Information. Romero, Reidy, Bootsma, PreFontaine, Vryhof, Wierenga, Anderson

\section{Compound 26c}
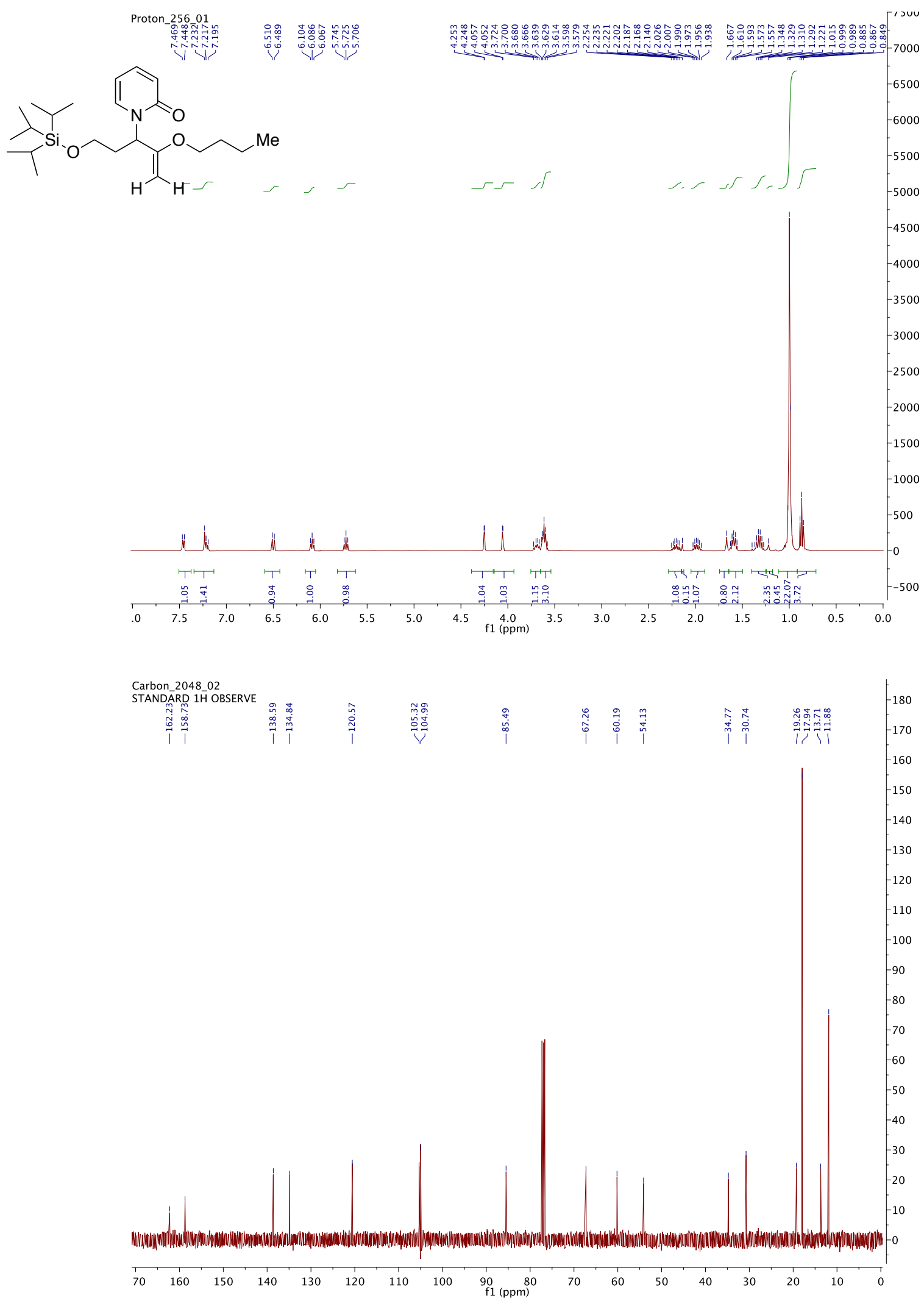
S17 Supporting Information. Romero, Reidy, Bootsma, PreFontaine, Vryhof, Wierenga, Anderson

\section{Compound $\boldsymbol{E}-27$}
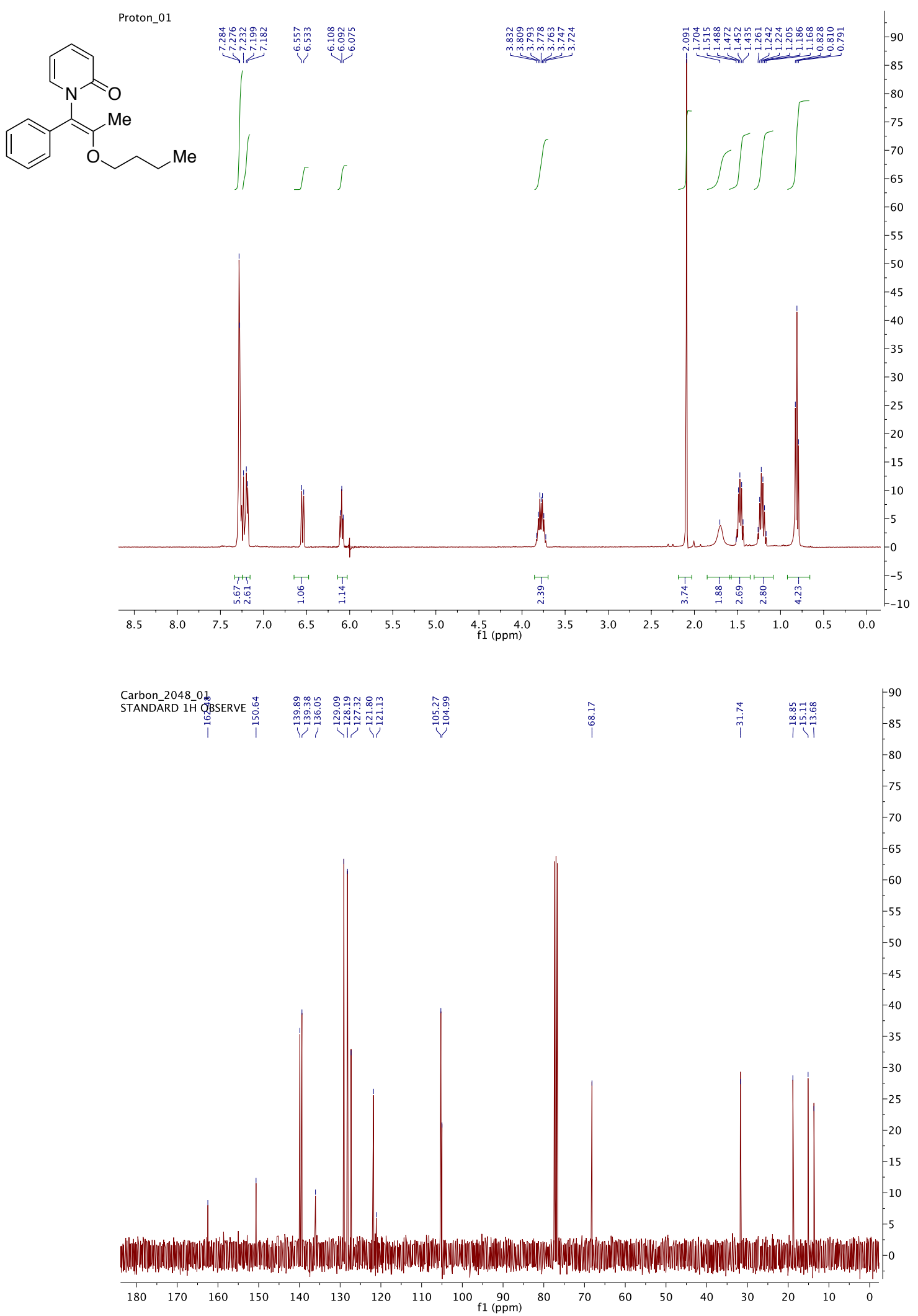
S18 Supporting Information. Romero, Reidy, Bootsma, PreFontaine, Vryhof, Wierenga, Anderson

\section{Compound $Z-27$}
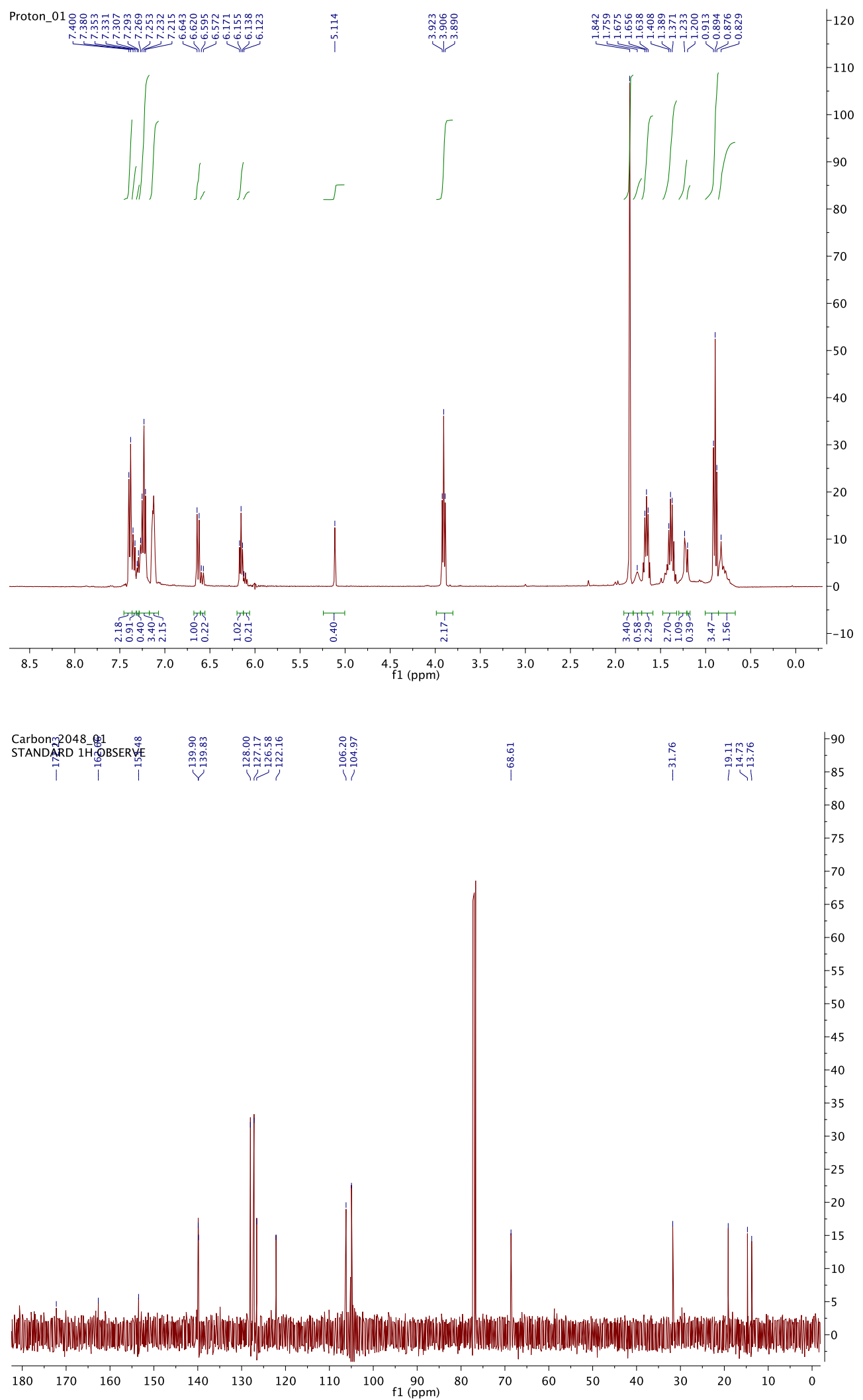
S19 Supporting Information. Romero, Reidy, Bootsma, PreFontaine, Vryhof, Wierenga, Anderson

\section{Compound 26f}
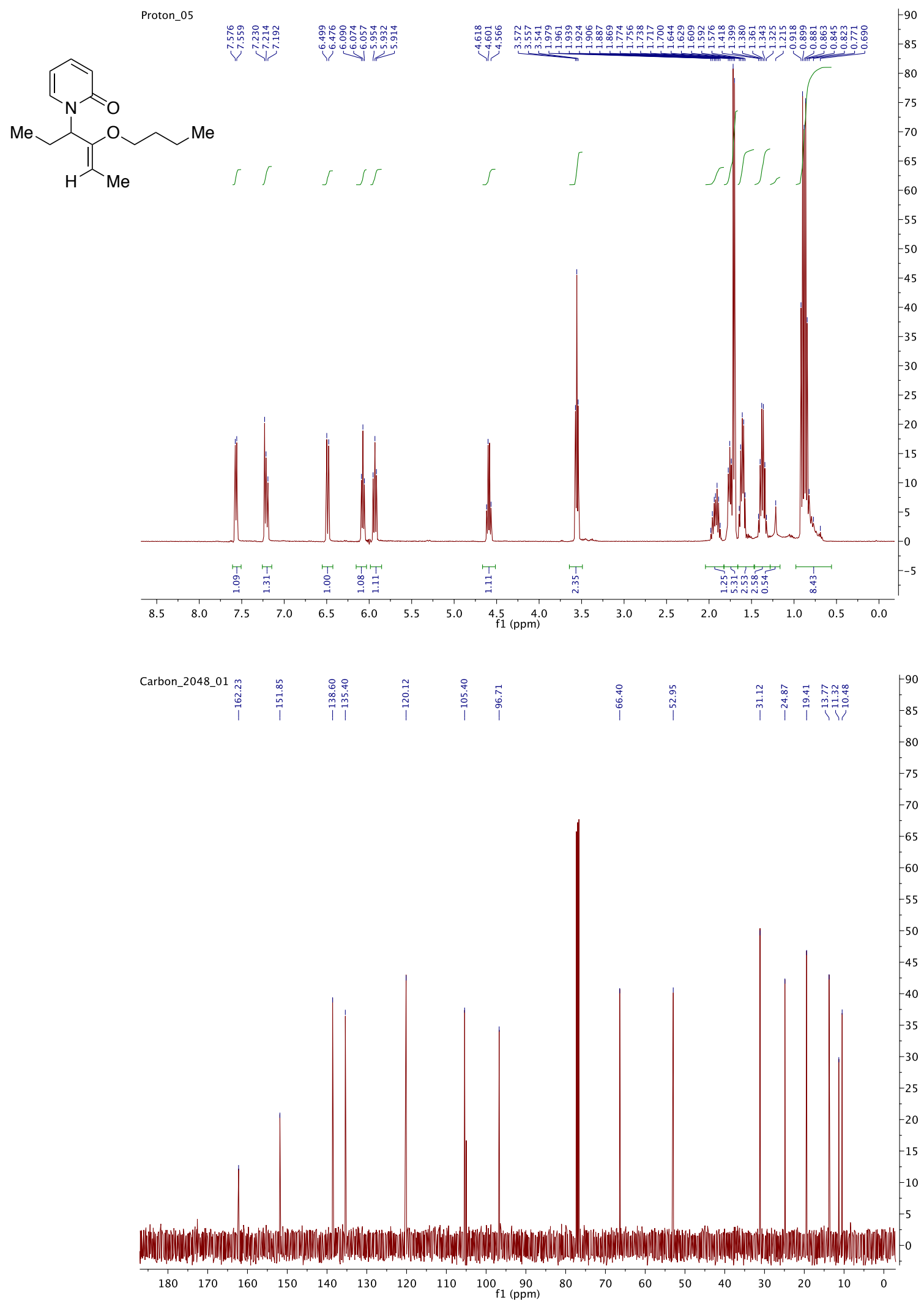
S20 Supporting Information. Romero, Reidy, Bootsma, PreFontaine, Vryhof, Wierenga, Anderson

\section{Compound 26g}
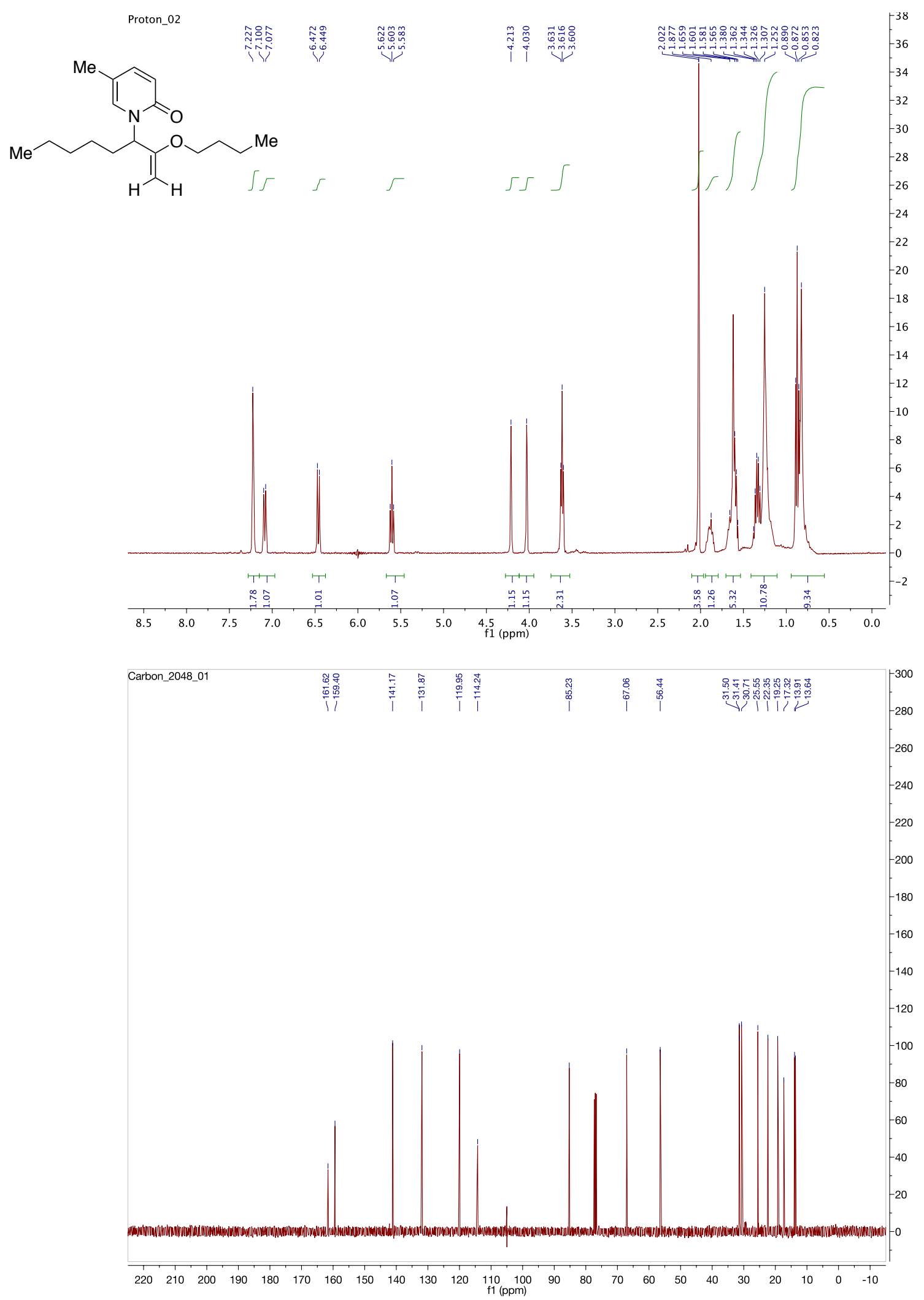
S21 Supporting Information. Romero, Reidy, Bootsma, PreFontaine, Vryhof, Wierenga, Anderson Compound 26h
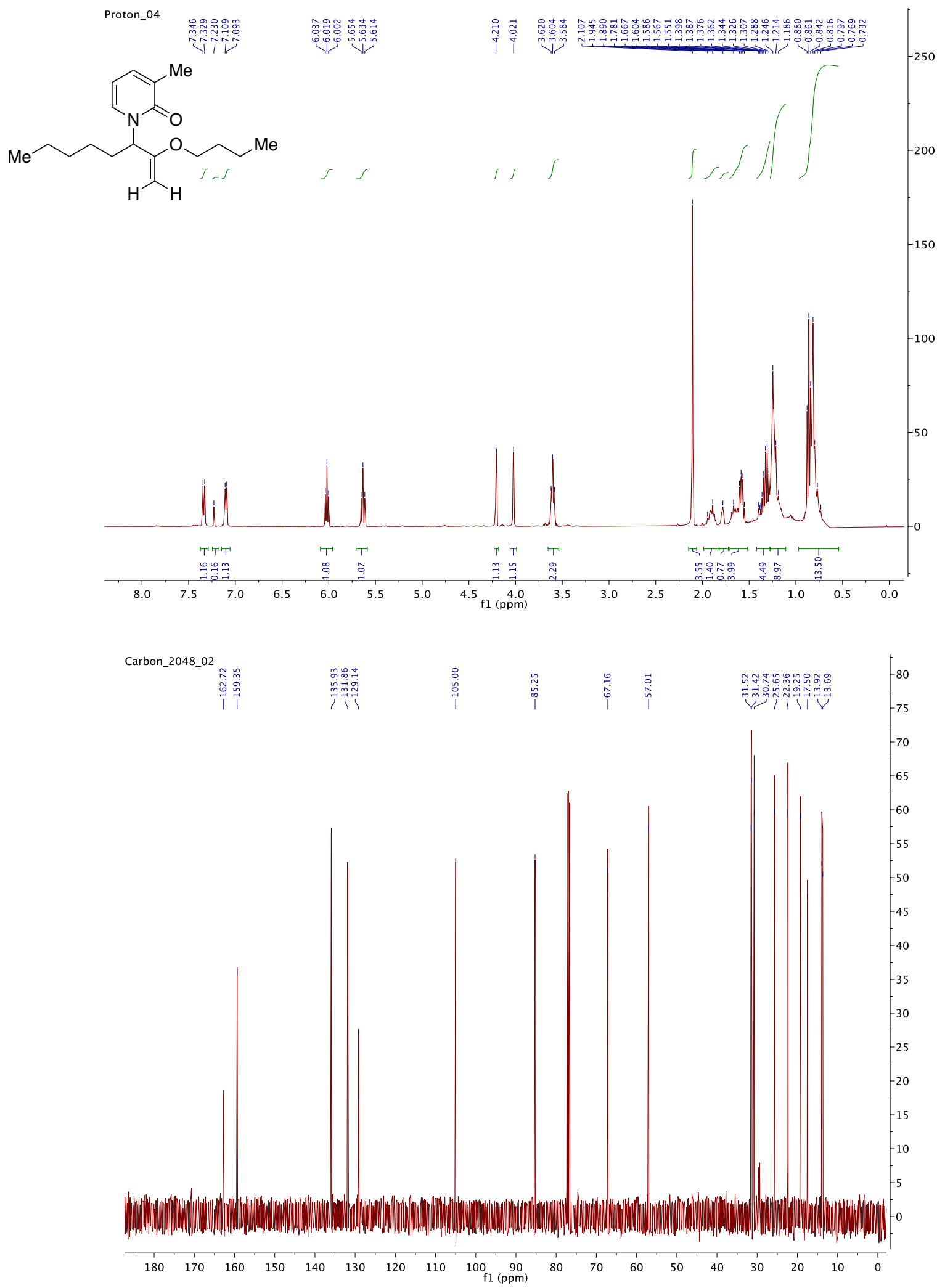
S22 Supporting Information. Romero, Reidy, Bootsma, PreFontaine, Vryhof, Wierenga, Anderson

\section{Compound 8b}
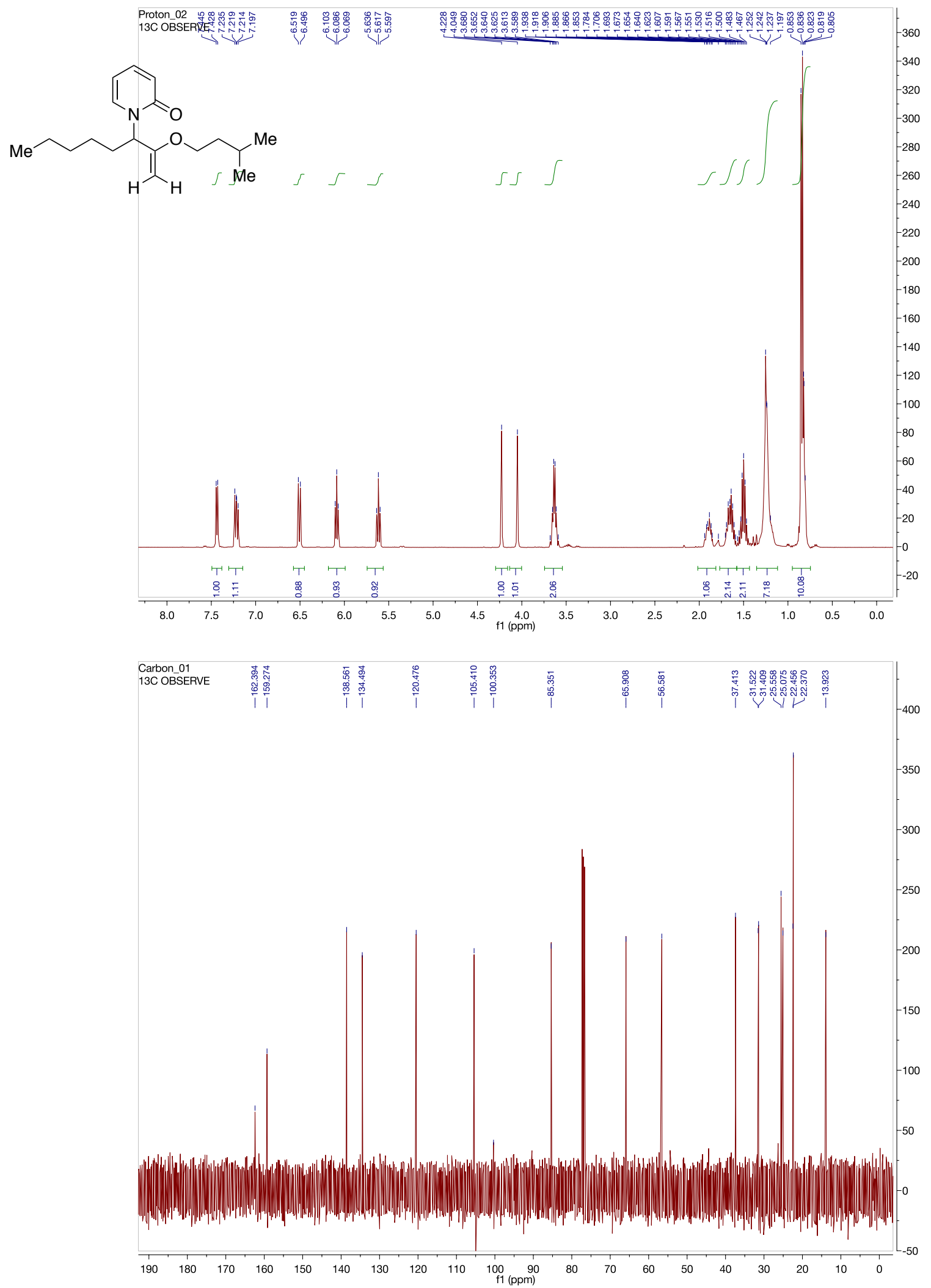
S23 Supporting Information. Romero, Reidy, Bootsma, PreFontaine, Vryhof, Wierenga, Anderson

\section{Compound 8c}
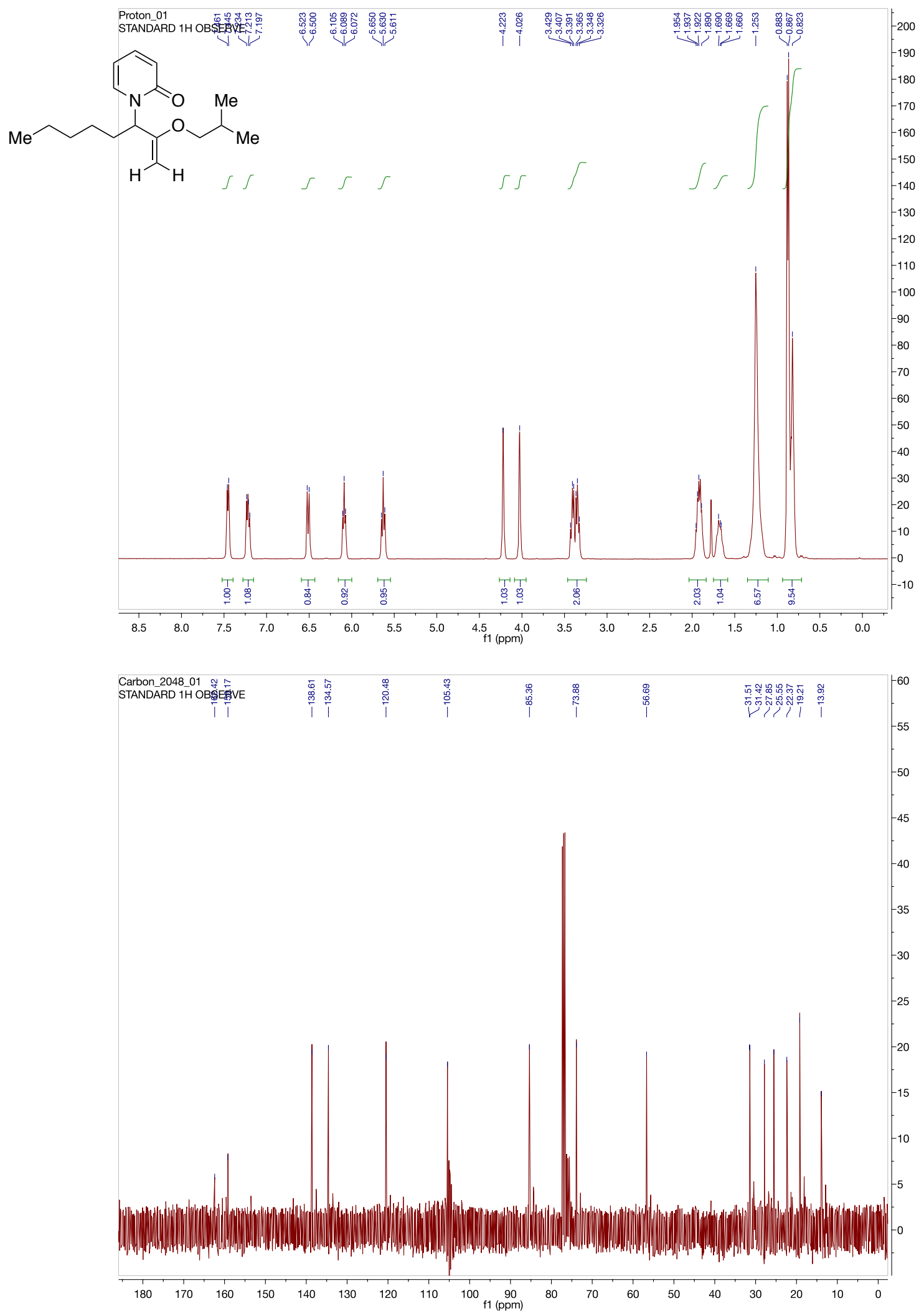
S24 Supporting Information. Romero, Reidy, Bootsma, PreFontaine, Vryhof, Wierenga, Anderson

\section{Compound 8d}
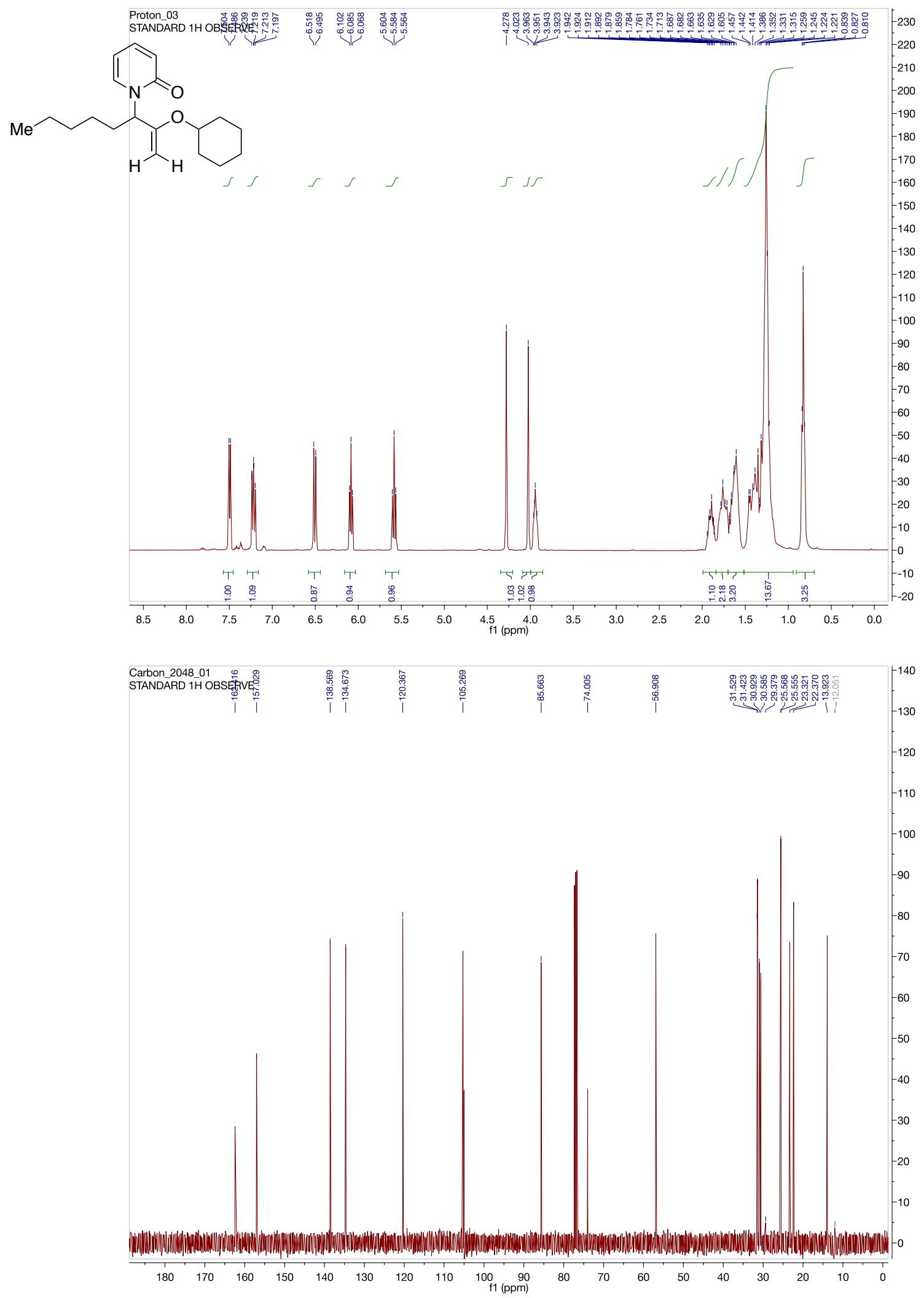